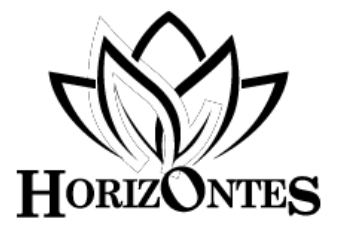

www.revistahorizontes.org
Horizontes. Revista de Investigación en Ciencias de la Educación Https://doi.org/10.33996/revistahorizontes.v4i13.92 Enero - Marzo 2020 Volumen 4 / No. 13 ISSN: $2616-7964$ pp. $64-83$ (c) (i) (\$) (2)

\title{
15 Años de las TIC en Investigación y Desarrollo Tecnológico de la UPEL
}

\section{Years of ICT in Research and Technological Development of UPEL}

\section{5 anos de TIC em pesquisa e desenvolvimento tecnológico da UPEL}

\author{
Belkys Juliana Guzmán \\ belkysguzman19@hotmail.com \\ belkys.juliana.guzman@gmail.com \\ https://orcid.org/0000-0002-8141-5990
}

Universidad Pedagógica Experimental Libertador. Instituto Pedagógico de Caracas, Venezuela

\section{Santiago Castro}

castrosantiago2015@gmail.com

https://orcid.org/0000-0003-2848-0870

Recibido octubre 2019 | Revisado nov - dic 2019| Publicado 01 de enero 2020

\section{RESUMEN}

Durante los últimos 15 años o más los productos académicos de la Universidad Pedagógica Experimental Libertador (UPEL), están relacionados con las Tecnologías de Información y Comunicación (TIC) y una problemática planteada en uno o más elementos del proceso educativo, en diferentes contextos, niveles o modalidades del sistema educativo. Este estudio pretende reconstruir los conocimientos y saberes relacionado con las TIC contemplados en los trabajos de grado, ponencias e investigaciones, especialmente los de investigación y desarrollo tecnológico de la UPEL. Es un estudio descriptivo que pretende explicitar la problemática, los contextos estudiados y que soluciones se han propuesto, con la intensión de hacer una línea de avances relacionados con las TIC en los procesos. Y hacer visible el conocimiento.

Palabras clave: Investigación y Desarrollo Tecnológico; Tecnología de Información y Comunicación

\section{ABSTRACT}

During the last 15 years or more, Universidad Pedagógica Experimental Libertador (UPEL) academic products are related to Information and Communication Technologies (ICT) and a problem posed in one or more elements of the educational process, in different contexts, levels or modalities of the educational system. This study aims to reconstruct the knowledge and knowledge related to ICTs contemplated in the degree, papers and research projects, especially those of UPEL research and development. It is a descriptive study that aims to explain the problem, the contexts studied and what solutions have been proposed, with the intention of making a line of advances related to ICT in the processes. And make knowledge visible.

Key words: Research and Technological Development; Information Technology and Communication 


\section{INTRODUCCIÓN}

Desde tiempos inmemoriales la tecnología está en nuestras vidas, desde antes de nacer y en la vida de nuestros antepasados, cambiando y haciendo más fácil nuestra vida. Las TIC conforma y tiene sus efectos sobre la cultura y por ende en los procesos de enseñanza y aprendizaje. (Guzmán, 2018).

En la actualidad la realización de transacciones bancarias de todo tipo tales como: transferencias, depósitos, pagos de tarjetas de crédito, procesos de admisión, asesorías, cursos, talleres, posgrados en Institutos y Universidades, hacer trámites de todo tipo: pagar los impuestos, sacar el Rif, hacer citas para obtener pasaportes o visas. Pago de bienes y servicios, Reservar y comprar; boletos de viaje en cualquier tipo de transporte, hoteles, restaurantes o simplemente leer la prensa o ver las transmisiones radiales. Es decir, estamos inmersos en el mundo y tecnológico que es parte de nuestro diario ir y venir.

La tecnología ha originado los cambios y transformaciones profundos en la sociedad el siglo XXI. La sociedad de conocimiento y de la información se desarrollan por la acción de las TIC que ha eliminado las barreras comunicacionales, ha impregnado de inmediatez a los dinamismos naturales $\mathrm{y}$ sociales.

La ciencia y la tecnología (Tecnociencia) como construcciones humanas y sociales conforman y son parte de la cultura de la sociedad, son lo que enmarca el hacer y el pensar del ciudadano y/o su grupo social. El ciudadano siente, piensa, oye, opina y actúa así edifica una institución, una nación o u planeta más equitativo, amigable y apto para la vida de él y sus congéneres, promueve la participación, la innovación, el emprendimiento y la satisfacción. Los desarrollos tecnológicos guían y determinan las políticas, la economía, la educación y por tanto la forma de vida de la ciudadana y del estado.

En la actualidad los saberes y conocimientos provenientes de la tecnociencia definida como la habilidad, destreza y disposición para utilizar adecuadamente los conocimientos, saberes y las metodologías usadas en el campo de la ciencia para explicar la naturaleza, así como su aplicación para modificar el entorno en respuesta a deseos o necesidades humanas (Álvarez, Arias, Pérez y Serrallé, 2013). Son el indicador de progreso, lo que marca y ha marcado la diferencia entre estados ricos y pobres.

La interacción del ser humano con las innovaciones producidas por el desarrollo tecnológico definido por Szczurek (1990) como "el puente entre lo descubierto por la ciencia y el usuario directo de esta", ha permitido que se integren las economías mundiales, al poner en contacto y modificar o por lo menos intervenir las diferentes culturas, en las que los grandes movimientos de capital y el comercio exterior demandan de personal altamente capacitado con competencias necesarias para tener movilidad y mayor incorporación al mercado productivo, con opciones válidas de empleo; esto ha generado una serie de necesidades que deben ser cubiertas, introduciendo los cambios que se requieran en el sistema (Guzmán, 2005, 2008).

La Investigación y Desarrollo Tecnológico ha provocado $\mathrm{y}$ provoca cambios $\mathrm{y}$ transformaciones en la naturaleza social, política, económica y cultural. Las estadísticas sobre el número $\mathrm{y}$ entidad de las organizaciones que se dedican a Investigación y Desarrollo Tecnológico (I+D o I\&D) pueden revelar el estado de la industria en un país, el grado de competencia o el progreso científico de una nación, pero ¿cuál es la estadística en el ámbito educativo? 
El desarrollo tecnológico y la innovación (I+D+I) como motores del desarrollo humano, social y económico para América Latina y el Caribe, es uno de los ejes temáticos de la III Conferencia Regional de Educación Superior para América Latina y el Caribe que considera entre sus objetivos:

1. Generar conocimientos y democratizar su acceso, uso y aprovechamiento como recurso común y bien público.

2. Fomentar el desarrollo de las Tecnologías de Información y Comunicación sin que se atente contra el derecho individual a la privacidad de los seres humanos.

3. Fomentar la apropiación social de la ciencia, las tecnologías y los conocimientos

4. Estimular activamente la vinculación estrecha de los ámbitos de investigación científica y tecnológica con las realidades y problemas de la vida social (productiva, ecológica, educativa y cultural), para promover soluciones innovadoras $\mathrm{y}$ factibles. (UNESCO, CRES 2018). Además, se debe agregar que en este mismo documento se señala que:

Los Estados y las instituciones de educación superior deben desarrollar estrategias de vinculación y programas colaborativos, a nivel de pre y postgrado garantizando los recursos para la innovación, la ciencia, la tecnología y la investigación, y así evitar la desvinculación entre la educación superior y el entorno productivo. (p. 17).

Algunos datos son: presupuestos dedicados Desarrollo Tecnológico, número de patentes, de prototipos electrónicos o no, o número de artículos basados en desarrollos realizados en empresas de capacitación o entrenamiento o escuelas, artículos de I+D+I publicados en revistas o puntos centrales de ponencias en eventos científicos. Los trabajos I+D+I buscan la excelencia, la satisfacción del cliente es su propósito primordial, su política de calidad es la mejora de los resultados y procedimientos para conseguir productos $\mathrm{y}$ servicios que incorporen el nivel de calidad esperado, en el tiempo deseado y dentro de los de los objetivos de coste fijados. Comprende los cambios introducidos en los sistemas e intenta proponer soluciones nuevas a los problemas. Se debe acotar que es necesario que todo desarrollador para hacer sus trabajos, tenga pensamiento sistémico lo que implica:
observar la relación entre las distintas partes expresadas en términos de retroalimentación, positiva o negativa, y en buscar el modo correcto, y no siempre obvio, de influir en el sistema en función de su eficiencia global. Implica pensar (o repensar)... en términos dinámicos en vez de estáticos, no lineales en vez de lineales y estructurales en vez de funcionales (Monzó, 2006 en Castro, 2009 p 173).

Durante los últimos 15 años los trabajos de desarrollo tecnológico educativo abordan temáticas diversas en diferentes contextos: escuelas, liceos, universidades en ellos han determinado necesidades: sentidas, normadas o detectadas en el rol de los estudiantes o praxis docentes de diferentes niveles 0 modalidades $\mathrm{u}$ otras profesiones como músicos, bibliotecarios, en las estrategias, medios y recursos e incluso en los elementos directrices y evaluación de los aprendizajes relacionadas con el uso y la apropiación de las TIC.

De esos trabajos han surgido distintas propuestas educativas que tratan de solucionar uno o varios de los problemas 
detectados, en este artículo describiremos algunas de estas soluciones que si bien no son extrapolables pueden servir de guía para abordar y aprovechar las potencialidades y características que ofrecen las TIC a la educación haciendo más efectivos y eficientes los procesos de enseñanza y aprendizaje, generando nuevas estrategias, medios $\mathrm{y}$ recursos, soluciones innovadoras en cualesquiera de las modalidades presencial, mixta o a distancia.

El propósito de este artículo es reconstruir la información relacionada con las Tecnologías de Información y Comunicación a partir de los estudios de desarrollo tecnológico educativo realizados en la Universidad Pedagógica Experimental Libertador en él se pretende específicamente:

1. Interpretar los conocimientos explícitos de los trabajos $y$ tesis de grado producidas en la UPEL-IPC en relación con las Tecnologías de Información y Comunicación.

2. Determinar la problemática relacionada con las TIC que se han estudiado en los últimos 15 años en la UPEL-IPC.

3. Describir algunas de las alternativas de solución planteadas.

\section{Recordando lo que es desarrollo tecnológico instruccional}

El impacto del desarrollo tecnológico y la innovación tecnológica en la sociedad en ámbitos educativos, políticos, económicos y socioculturales es innegable a como también lo son los beneficios que producen para aquellas personas que las manejan $y$ aprovechan sus potencialidades. Los cambios afectan a los ciudadanos y a toda la sociedad en su diario ir y venir, estos cambios exigen nuevas competencias que le permiten vivir, movilidad, hacer y ser un activo valioso dentro de este entorno cambiante.
Lo que crea en él nuevas necesidades de autorrealización, capacitación y formación, el reto del sistema educativo es adaptarse y asumir su función asumir los cambios e introducir las mejoras requeridas. Para hacer esto posible se debe echar mano de las TIC sus características y potencialidad. Pero ¿qué es I+D o I\&D? cuáles son las etapas de ejecución se comenzará por señalar que Colciencias (1999 s/p) considera que el:

Desarrollo Tecnológico busca
asimilar, mejorar, adaptar o
cambiar la forma de hacer las
cosas con base en la aplicación de
tecnologías diferentes a las que se
han venido utilizando, en un
campo específico de aplicación,
con el fin de mejorar la
productividad, la competitividad
y la rentabilidad de la
organización.

Mientras que Moreno Posada, (1978) define el desarrollo tecnológico educativo como:

La actividad emprendida con el fin de utilizar los resultados que se han obtenido en la investigación básica y aplicada, incluyendo los conocimientos empíricos en el campo instruccional, con el fin de introducir nuevos materiales, procesos, métodos, productos, dispositivos, equipos y sistemas operativos, o mejorar los ya existentes, incluyendo la constitución y prueba de prototipo o instalaciones experimentales.

Los objetivos y productos de la investigación y el desarrollo tecnológico son diferentes, no es suficiente el proceso de investigación es necesario completar el ciclo con la introducción exitosa de sus resultados 
al mercado o a la sociedad, es decir, la suma de concepción, la idea, el problema, la invención y explotación para realmente hacer efectiva la solución.

La comunidad universitaria está poco o desinformada sobre el D+I, por lo que generalmente piensan que el desarrollo tecnológico está ligado exclusivamente al uso de materiales y equipos informáticos. Mientras que Escorsa y Valls (2001), plantean que es una acción humana e involucra procesos intelectuales, se refieren a relaciones causales dentro del mundo real y, en algunos casos, emplean metodologías experimentales de las cuales surgen demostraciones empíricas que pueden verificarse. En D+I se usa el conocimiento y el saber para la producción de materiales, dispositivos, procedimientos, procesos, sistemas o servicios nuevos o mejoras sustanciales originando ensayos y pruebas de prototipo o pruebas piloto. Puesta en marcha de una solución a un problema práctico, en nuestro caso de un sistema instruccional.

Szczurek escribe que: (comunicación personal, correo e, octubre 25, 2006):

el uso de los resultados de la investigación es del dominio del Desarrollo Tecnológico, que no de la Investigación Aplicada. Ciertamente ese dominio no es sólo el diseño (instruccional o de lo que sea), sino también la previa determinación del problema en sí y su contexto, y la posterior implantación y determinación del grado de logro de la solución.

\section{Modelo general de desarrollo tecnológico y cómo se desarrolla cada etapa}

Hay varios modelos que presentan pautas a seguir para realizar los trabajos de desarrollo tales como los propuestos por:
Colciencias, (1999 nov), Buch, (1996), Kaufman y Stone. (1987), Chadwick. (1987 p31) y Müller y Szczurek (1989) todos coinciden en que son modelos sistémicos que parten de un estudio de necesidades y culmina en una evaluación del sistema. Por lo que en nuestra universidad desde hace 30 años decidimos utilizar la estructura para trabajar el I+D presentado por Müller y Szczurek (1989) a la primera cohorte de la Maestría en Educación mención Tecnología y Desarrollo de la Instrucción de la UPEL de la cual uno de los autores es egresada, luego exhibido por Szczurek (1990) en las jornadas de Tecnología Educativa, y luego discutido y analizado por Zabatta, en su artículo publicado en 2008 en el cual se describe algunas reflexiones acerca del modelo.

El Modelo General de Desarrollo Tecnológico propuesto por Szczurek (1990) presenta varias fases o etapas que son insumo para la toma de decisiones y realización de la siguiente. Estas fases incluyen procedimientos lógicos, coherentes y conexos que fundamenta un conjunto de acciones sustentadas en el estudio y los resultados generando en cada etapa brindan aportes sólidos para trazar las distintas orientaciones que apunten ofrecer una ingeniería en los distintos entornos $\mathrm{y}$ contextos sociales abordados (Quintero J. Yoli, 2017).

Este modelo es usado explícitamente en todos los trabajos de grado culminados con éxito en la UPEL en las maestrías: en a) Educación mención Tecnología y desarrollo de la Instrucción, b) enseñanza de la Biología, c) enseñanza de la Química, d) maestría en Educación Ambiental, así como también en diferentes ponencias. Castro y Guzmán (2001) señalan que el trabajo de Desarrollo Tecnológico Instruccional presenta cuatro etapas que son: Estudio de necesidades, Diseño instruccional, Simulación y/o Implantación y Evaluación. Como afirma 
Müller (2003 p141) "cada una de las etapas están colocadas de forma que sus productos sean insumos para la elaboración de la siguiente".

Según Quintero J. Yoly (2017) las fases propuestas por Szczurek (1990) corresponden a: (a) levantamiento y análisis de los hallazgos claves que permiten la comprensión de las distintas interrelaciones que se suscitan en el ambiente de estudio, los cuales se sustraen a través de la aplicación de modelos, estrategias, medios, técnicas herramientas e instrumentación adecuadas, (b) la construcción de un diseño basado en los hallazgos obtenidos y elementos intervinientes. Este buscará atender y subsanar el o los problemas presentes en cada ambiente, (c) la implantación o puesta en práctica del plan de solución diseñado en la fase anterior y (d) las valoraciones y los resultados del proceso de implantación, que permiten ejercer modificaciones pertinentes.

Es importante destacar que los elementos evaluativos se evidencian no solamente en el producto de la implantación, sino que aborda de manera transversal todas y cada una de las etapas que dicho modelo sistémico, atiende el estudio de necesidades consiste en diagnosticar las necesidades de la organización o ente intervenido, tomar decisiones en torno a cuáles se han de satisfacer y en qué medida, y proponer una solución. Este estudio como todo trabajo de investigación se inicia con inquietud enmarcada en una problemática mundial, nacional $\mathrm{y}$ en muchos casos regionales $\mathrm{e}$ institucionales.

Los autores de estos trabajos enfrentan el primer problema que es saber cómo abordar cada etapa, los modelos, pasos, técnicas e instrumentos adecuados a la hora de ejecutarlas, lo que puede conducir a un desgaste de tiempo, dinero, y baja en la motivación que en muchos casos puede llegar a la deserción, es por ello que se idearon estos esquemas que se desarrollan más adelante que ayudan a solucionar este problema.

\section{Estudio de necesidades}

Para el estudio de necesidades, primera fase del modelo, se documenta, caracteriza y justifica una necesidad de cambio o de innovación y genera las ideas de las posibles vías para realizarlo. Una vez detectadas las brechas instruccionales entre la situación real (o él es) y la situación ideal o deseada (debe ser) y seleccionadas las alternativas de solución factibles con mayores probabilidades de éxito, se procede a realizar el diseño instruccional correspondiente. En esta evaluación diagnóstica, para establecer el verdadero problema se pueden utilizar los enfoques cualitativos, cuantitativas o multi métodos que en función de los beneficiarios y los temas abordados. Del estudio de los trabajos analizados desde 1992 se desprende que para el estudio de necesidades se han usado Kaufman (1978) y Kaufman y Stone (1987) y posteriormente Müller (2003).

A continuación, se muestran 4 gráficos que presentan preguntas cuyas respuestas dan origen a la metodología a seguir para realizar la etapa, no es un modelo único, pero ayuda a desarrollar cada una con de ellas con certeza de que se están siguiendo todos los pasos correspondientes. En todos ellos hay 5 preguntas claves cuyas respuestas le permiten al autor la guía necesaria para el desarrollo de su trabajo. En el grafico 1 que explicita la información que hay que considerar en relación con esta etapa evaluativa.

Hay personas que consideran que para la realización del estudio de necesidades se debe hacer en forma cuantitativa con la única excusa de que se sabría exactamente el porcentaje de brecha que hay entre el es y el debe ser. Pero esta no es un criterio adecuado 
para seleccionar el método de investigación a utilizar existen criterios más validos que este. Si el problema a tratar es netamente social y se obtiene una información en función del reflejo de las experiencias vividas, los comportamientos, emociones y sentimientos de un grupo de informantes entonces tenemos un diagnóstico basado en un análisis cualitativo $\mathrm{y}$ es tan valioso, objetivo $\mathrm{y}$ pertinente como lo es el cuantitativo.

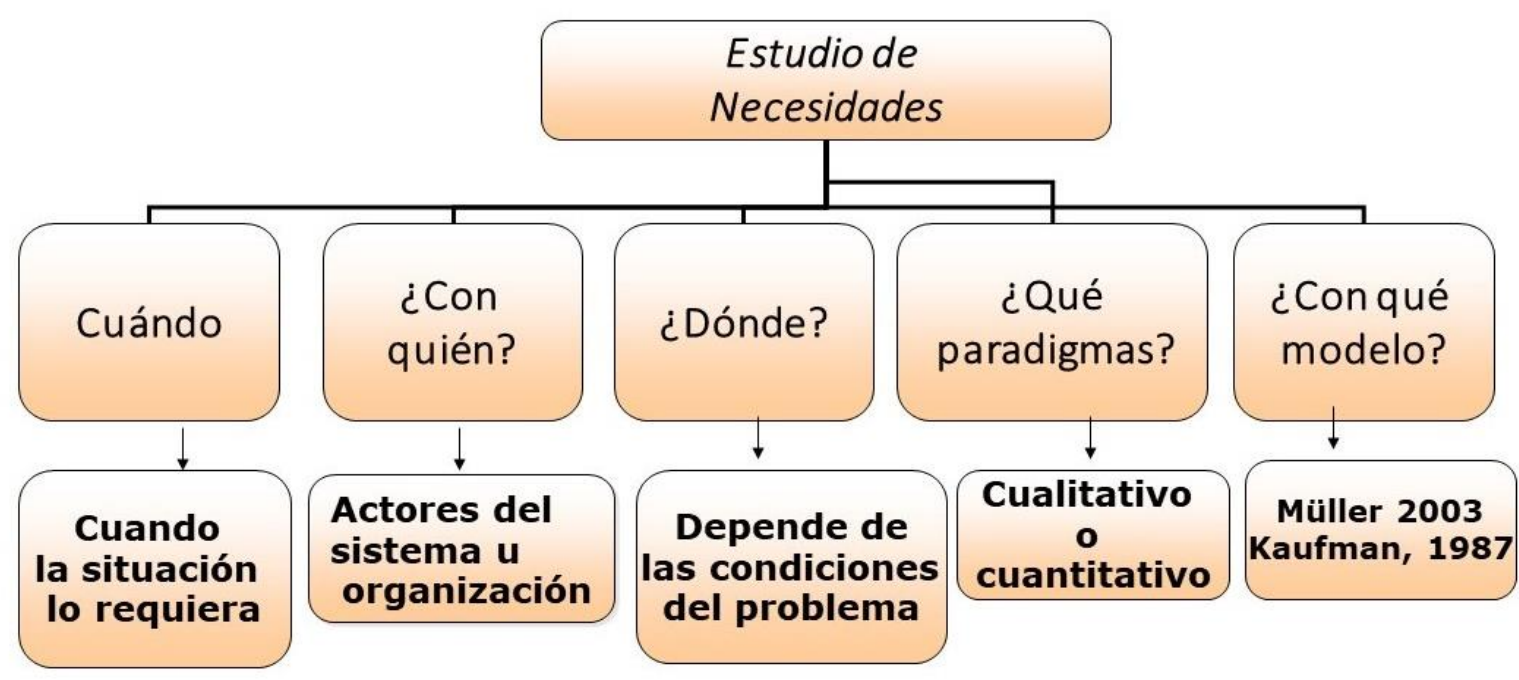

Grafico 1. Estudio de Necesidades Tomado de Castro y Guzmán, (2012) materiales del curso de Tutoría de la maestría en TDI.

El gráfico, que habla por sí solo señala que el Estudio de Necesidades da una base clara para generar las alternativas de solución planteadas para mejorar o solucionar el problema detectado, en consecuencia, deben ser sometidas a un estudio de factibilidad. Es un instrumento que sirve para orientar la evaluación del proyecto y que da base para tomar decisiones en cuanto a su simulación, implantación o no.

\section{Diseño instruccional}

El diseño instruccional la segunda etapa referida en todos y cada uno de los trabajos de desarrollo tecnológico realizados en esta maestría desde su creación vienen a constituir la materialización en términos prácticos de las teorías de la Instrucción con ellos se abren múltiples perspectivas de creación, diseño, y desarrollo de materiales. El diseño instruccional se convierte en múltiple, variado y holístico. En él se genera una diversidad de interacciones que deben ser integradas en los procesos de enseñanza y aprendizaje Se estimula el esfuerzo personal y grupal mediante escenarios que tienden a resolver problemas. Se facilita el acceso a la información a través de debates generadores de conocimientos, mediante la discusión dentro de grupos de trabajos (Stojanovic, 2009).

Los Diseños Instruccionales deben perfilarse bajo una nueva visión de mundo y del conocimiento, tendente hacia construcciones socializadas con carácter interdisciplinario. En consecuencia, es innegable que las instituciones de educación superior están llamadas a conjugar esfuerzos para enfrentar tal desafío en la formación de futuras generaciones para la actual sociedad del conocimiento. 
Los Diseños Instruccionales apoyados en las TIC pueden ser clasificados de acuerdo con un propósito o alcance en dos grupos: (1) los diseños centrados en el profesor, orientados a la enseñanza de contenidos, en cuyo caso representa en el ciberespacio el modelo de clases tradicional; y (2) diseños emergentes orientados, además de la enseñanza del contenido curricular, al desarrollo de estrategias cognitivas para el aprendizaje autónomo y permanente. (C. Ruíz Bolívar, conversatorio personal, enero 15, 2007) citado por Oviedo 2010.
Los factores primordiales para la escogencia de un modelo de diseño son además de las características y necesidades de los usuarios, las teorías de enseñanza y aprendizaje y los estilos de aprendizaje e inteligencias múltiples.

El gráfico 2 que se muestra a continuación explicita la información que hay que considerar en relación con esta segunda etapa del modelo de $\mathrm{D}+\mathrm{I}$ propuesto.

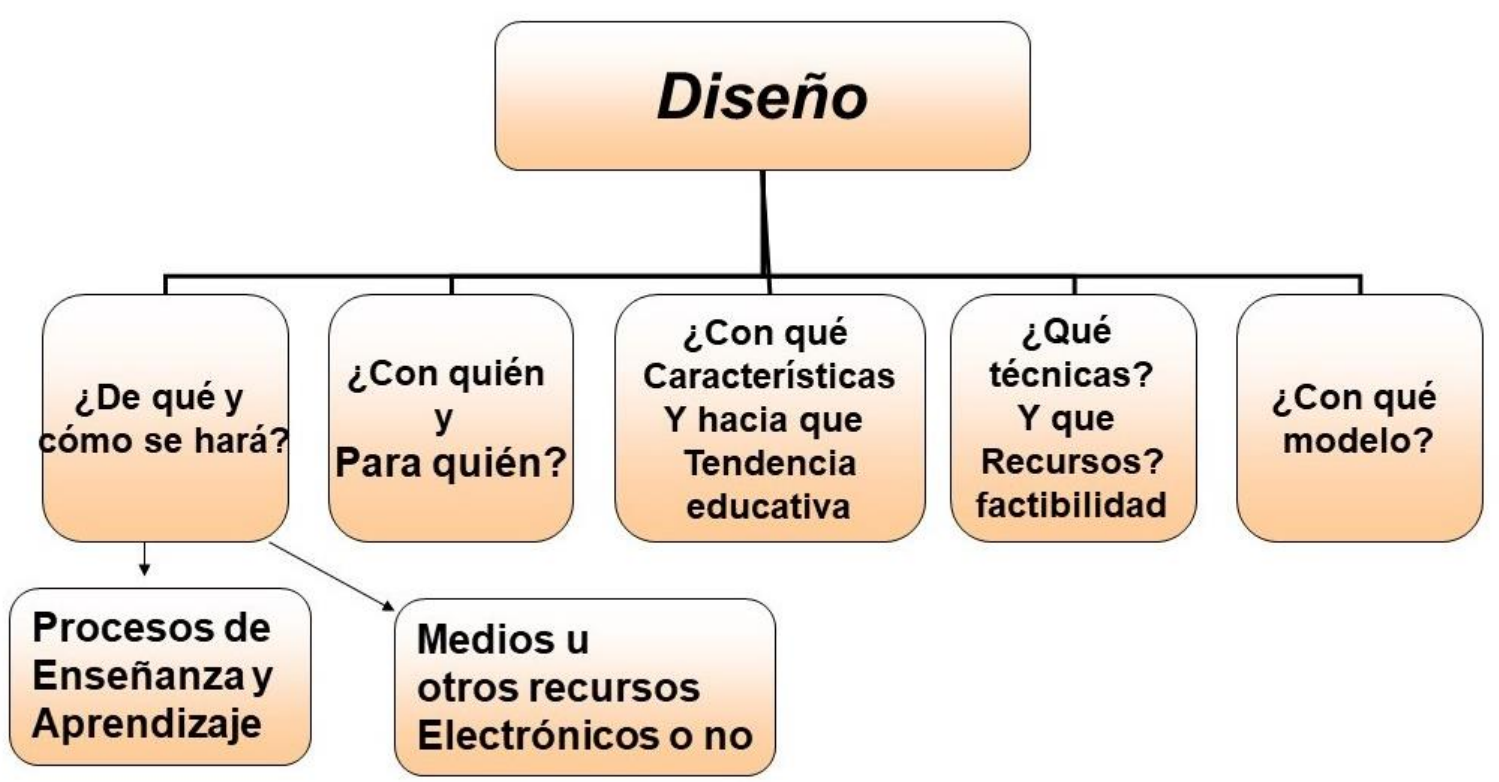

Grafico 2. Diseño de Instrucción. Tomado de Castro y Guzmán, (2012) materiales del curso de Tutoría de la maestría en TDI

El grafico muestra que la decisión de cuál o cuáles modelos se deben usar para el diseño de la solución va a depender del tipo de solución planteada, si esta es cognitiva, afectiva o motora y también si se trata un taller un curso o un material instruccional. Es importante señalar que los medios y recursos pueden ser parte de la solución cuando forman parte de un curso - taller $y$ dependiendo de la audiencia pueden ser la solución planteada a la necesidad. Cabe acotar que los trabajos de desarrollo tecnológico revisados muestran diseños instruccionales que responden a diferentes teorías de aprendizaje que van desde el conductismo, cognitivo, constructivistas o conectivistas este último considera que "el aprendizaje no es un proceso lineal sino reticular, y que debemos adaptarnos a esta era de la información masiva, instantánea y cambiante" (Siamens en Solano 2017). 


\section{Simulación y/o Implantación}

Szczurek (1990) propone que la tercera etapa la constituye la simulación y/o implantación que representan en todo sistema instruccional uno de los componentes potenciales que ejercen influencia sobre el aprendizaje del estudiante, uno de ellos es la planificación, el otro es la transmisión de la instrucción, es decir, la puesta en práctica de las actividades planificadas, punto que corresponde tratar ahora.

La simulación consiste en el empleo de un modelo de un suceso o situación verdadera o prevista con el objeto de ver cómo funciona en la práctica. Para GÖptepe, Özgüc, y Baray, (1989) es una representación controlada de fenómenos del mundo real, mientras que Seidner (1976) la considera como una modelación de realidad social o física de manera que el participante pueda interactuar y llegar a ser parte de esa realidad simulada generalmente se utiliza cuando la situación es riesgosa o la población es muy grande.

En esta etapa de implantación se evalúa y toman decisiones para aumentar la eficacia y efectividad de un método o un medio (Kaufman, 1983). La implantación equivale a hacer realmente lo que se planificó, utilizando las estrategias e instrumentos seleccionados en poblaciones pequeñas o de poco riesgo. Se ejecutan los planes, evalúan los materiales a usar, se contrata o prepara a determinadas personas para aplicar lo planificado o recoger la información. Esta etapa puede administrarse y controlarse de modo que se introduzcan los correctivos necesarios y se logren los resultados previstos (Kaufman, 1983).

El gráfico 3 que se muestra a continuación explicita la información que hay que considerar en relación con esta etapa.

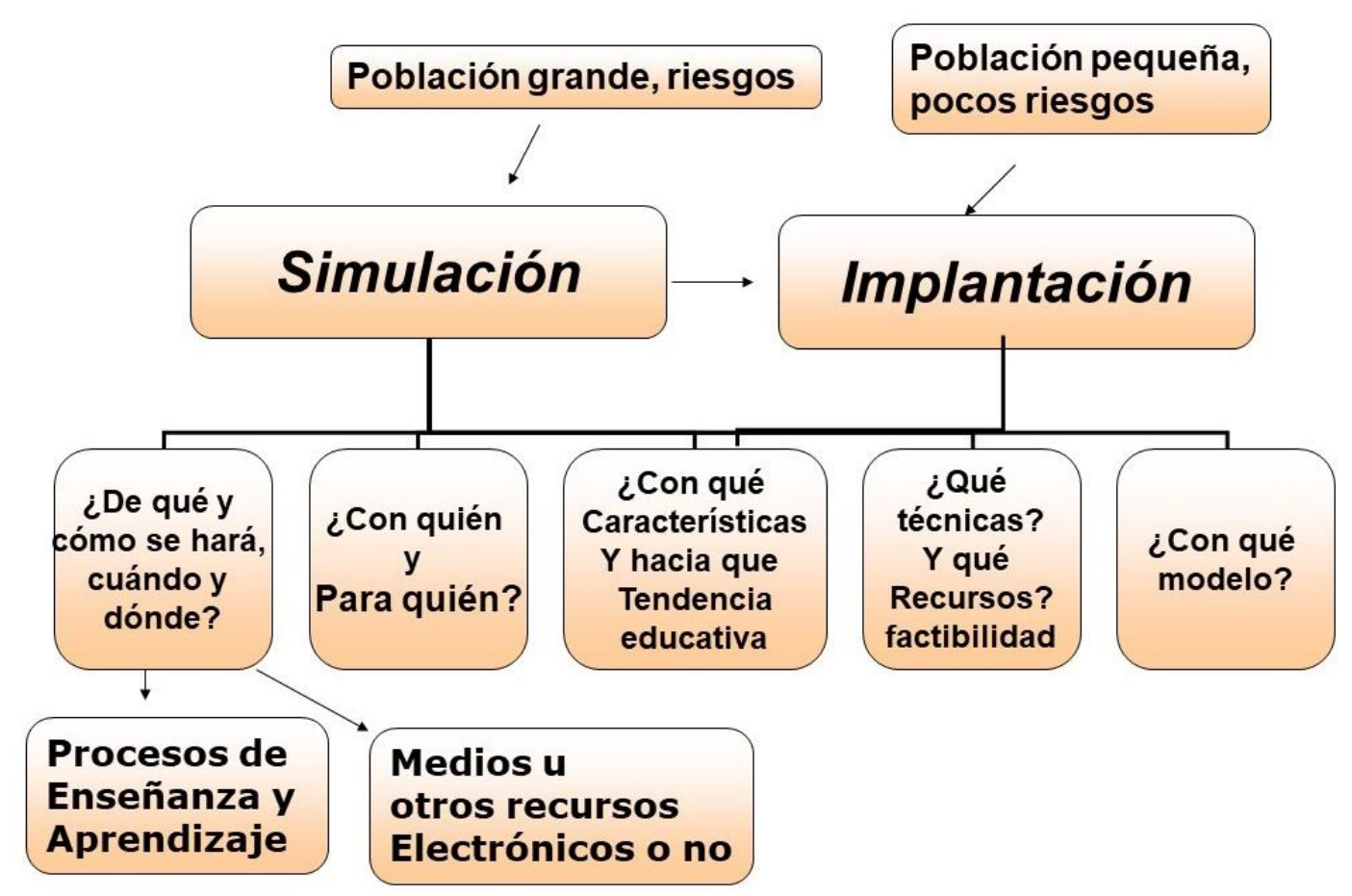

Grafico 3. Simulación y/o Implantación Tomado de Castro y Guzmán, 2012 materiales del curso de Tutoría de la maestría en TDI 
La última etapa, aparente, pero no menos importante la constituye la evaluación del sistema estudiado es un componente y no un proceso separado sin relación con los demás elementos del sistema. Esta concepción de la evaluación permite poner en práctica un análisis centrado, tanto en los aspectos internos del programa y el logro de sus objetivos por los aprendices, como en la relación entre el programa y el contexto del cual forma parte (Peñaloza en Castro 1993). El gráfico 4 que se muestra a continuación explicita la información que hay que considerar en relación con esta etapa.

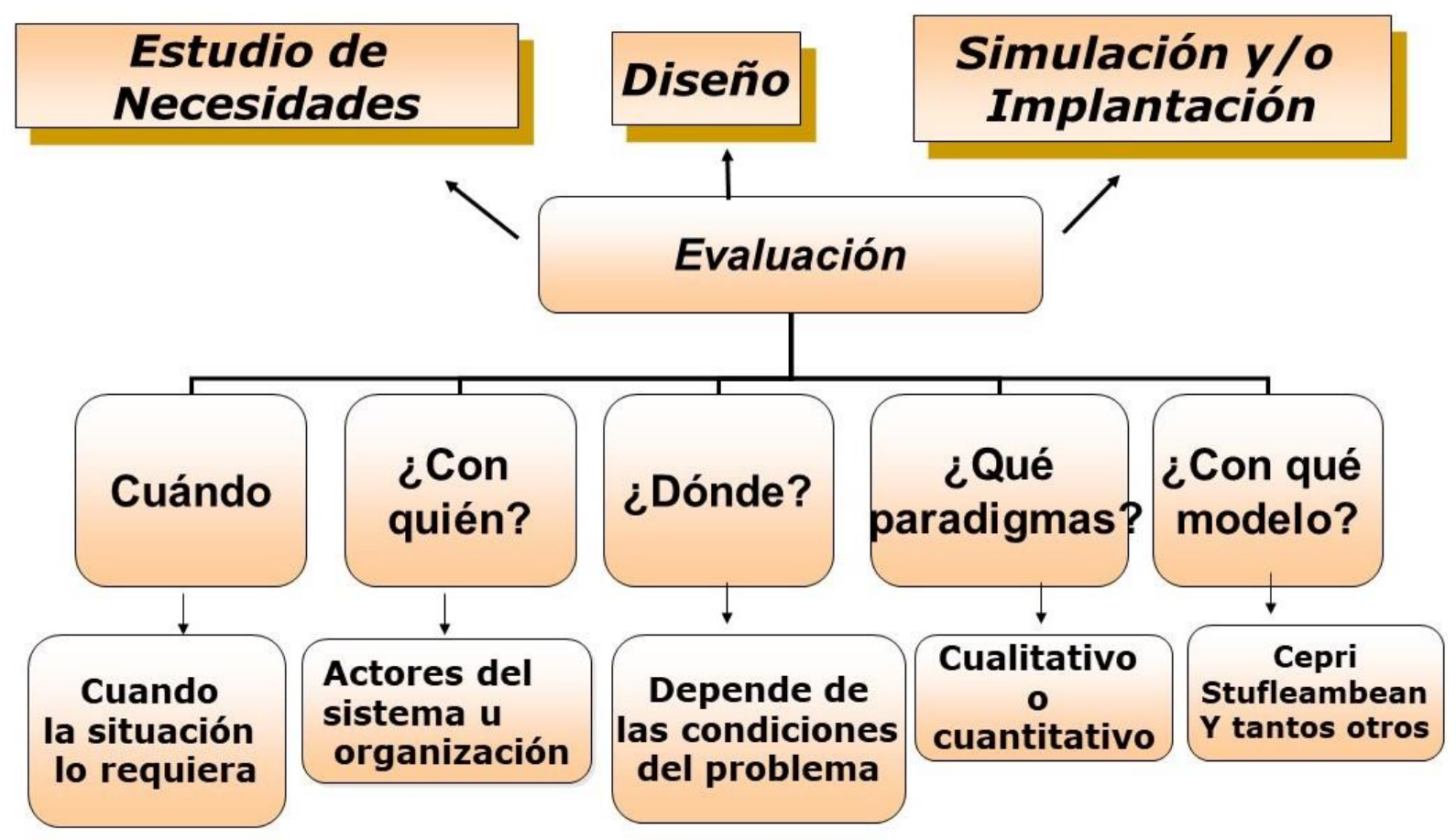

Gráfico 4. Evaluación Tomado de Castro y Guzmán, 2012 materiales del curso de Tutoría de la maestría en TDI

Es de acotar que se evalúan todas las etapas del sistema el contexto, la entrada, el proceso, el producto, es decir se evalúan las necesidades, el diseño la simulación e implantación incluso la evaluación del mismo. La realización de todas estas etapas conduce al desarrollo de experiencias creativas, innovadoras y de evaluación como mediadores para la transformación del ámbito escolar, acciones para optimizar los procesos de enseñanza y aprendizaje y solución de problemas en diferentes ámbitos educativos $\mathrm{y}$ organizacionales. Con el desarrollo de planes, proyectos y programas de intervención para mejorar el rendimiento integral de diversos procesos educativos. Programas de intervención en los roles de los actores del sistema educativo y sus características, inteligencias múltiples, estilos de aprendizaje, autorregulación del aprendizaje. Entre otros. ¿Hemos hablado de DTI, pero y las TIC que ha sido su objeto de estudio? 


\section{Tecnologías de la información y la comunicación}

Desde hace algunos años el tema más estudiado de los trabajos son las tecnologías de la información y la comunicación (TIC) estas adquieren mayor peso y están más presentes en las vidas, y afecta a las relaciones interpersonales y con el entorno, estando también presentes en los procesos de enseñanza y aprendizaje. En estos trabajos asumen los conceptos, características y muchos de las asunciones que se muestran a continuación.

Las Tecnologías de Información y Comunicación están cada vez más presentes en todos los ámbitos de nuestras vidas. Este avance afecta a las emociones, las relaciones intra e interpersonales y por ende al contexto, por lo que las tecnologías están imbricadas en los procesos de enseñanza y aprendizaje. Todos los actores del sistema educativo han visto cómo la tecnología digital se implantó en su diario ir y venir, por lo que hace imperioso su apropiación. En la UPEL y en este contexto país tenemos un punto de inflexión: entre la inmersión en la tecnología y la formación continua y permanente que va a requerir los elementos del sistema de enseñanza $y$ aprendizaje para seguirle el ritmo a los avances científicos-tecnológicos tanto dentro como fuera del aula.

Guzmán (2005, 2008) asume las siguientes definiciones de las TIC planteada por Bartolomé $(1989,11)$ como los últimos desarrollos tecnológicos y sus aplicaciones. En esta misma línea en el diccionario de Santillana de Tecnología Educativa (1991), las definen como los "últimos desarrollos de la tecnología de la información que en nuestros días se caracterizan por su constante innovación." Como se puede observar seguimos hablando de DTI, revisando otros conceptos tenemos que Cabero (1996 p-4) expresa: $\begin{array}{lrrr}\text { Gilbert y } & \text { otros } & (1992,1), & \text { hacen } \\ \text { referencia al "conjunto de }\end{array}$ herramientas, soportes y canales para el tratamiento y acceso a la información". "Castells y otros (1986) indican que comprenden una serie de aplicaciones de descubrimiento científico cuyo núcleo central consiste en una capacidad cada vez mayor de tratamiento de la información". Y por último el concepto publicado en la revista "Cultura y Nuevas Tecnologías" de la Exposición Procesos, que lo define como "... nuevos soportes y canales para dar forma, registrar, almacenar y difundir contenidos informacionales." (Ministerio de Cultura, 1986, p. 12).

Guzmán, (2005, 2008, 2013, 2018; Castro, Casado, y Guzmán. 2007) refieren que las TIC:

Es el conjunto de sistemas y productos que se materializa mediante dispositivos informáticos y de interconexión que funcionan por medio de programas que las personas usan para llevar a cabo procesos de tratamiento de información y de comunicación". Estos avances tecnológicos varían en atención a los escenarios en los que las personas pueden compartir conocimientos, tal como lo presentan las diversas teorías de aprendizaje.

Las Tecnologías de la Información y Comunicación son herramientas que procesan, almacenan, sintetizan, recuperan y presentan información representada de la más variada forma. "Son medios colectivos para reunir, almacenar, transmitir, procesar y recuperar información electrónicamente, así como el control de toda especie de aparatos de uso cotidiano hasta las fabricas automatizadas" (Gerstein citado por Rebolloso, 2000). 
Mientras que Guzmán, (2013) señala que en los procesos de enseñanza y aprendizaje se construye entre docentes y estudiantes una relación empática creando un clima afectivo que permite al estudiante convertirse en el principal actor de su aprendizaje. La interacción entre los elementos del sistema es primordial para un desarrollo adecuado del curso, tema, taller o unidad curricular, un clima de aula agradable, un trabajo efectivo, así como también un ambiente promotor de la creatividad donde todos son respetuosos de sí mismos y de los demás y son tratados con justicia, equidad y democracia, permite mayor productividad con creatividad, originalidad y calidad.

Estas interacciones respetuosas entre los actores del sistema construyen una serie de códigos con características específicas de uno y otros que les amplia los canales de comunicación y por ende propician una mejor interacción entre ellos. Ahora bien, enseñar y aprender en la actualidad requiere, de docentes capaces de transformar su praxis pedagógica utilizando las TIC, no está sujeta a restricciones espaciales o temporales, para atender a sus estudiantes y enfrentar los múltiples desafíos que esto generan. Esto requiere la autorregulación del aprendizaje. La interactividad es la clave en la comunicación surge de hablar y escuchar, tanto cara a cara, como a través de dispositivos.

Guzmán, (2005, 2008) y Casado, Castro y Guzmán, (2007) consideran que las características de las TIC aplicadas a todos los elementos del sistema educativo, en cualesquiera de sus niveles o modalidades, sea distancia, presencial o mixta promueven el intercambio de papeles entre los actores de los procesos de enseñanza y aprendizaje, de estrategias, de medios y recursos, así como también de mensajes entre los diferentes actores del sistema instruccional: estudiantes, estudiantes - docente y estudiantes materiales, entes que consumen, producen y distribuyen información.

Con las TIC toda innovación logra abarcar tanto la idea de un desarrollo tecnológico como la de un cambio social. Da inicio con idea, nueva, valiosa, creativa que se ejecuta en una organización ya sea esta educativa, empresarial, comunitaria entre otras, por parte de individuos y equipos de trabajo. La innovación debe ser una cultura de cualquier organización con miras a la visión y misión de esta.

Las principales funciones de las Tecnologías de la Información y Comunicación en los trabajos formulados en los diferentes I+D están relacionadas con:

1. Alfabetización digital de los estudiantes, profesores u otros profesionales.

2. Uso y apropiación personal de las TIC para el acceso a la información, comunicación, gestión y procesamiento de datos.

3. Gestión del centro educativo, secretaría, biblioteca, laboratorios, gestión de la tutoría de estudiantes e investigadores.

4. Uso didáctico para mediar los procesos de enseñanza y aprendizaje.

5. Comunicación didáctica o no con familiares, amigos, o estudiantes.

6. Comunicación interpersonal con personas del entorno cercano o no.

7. Desarrollo de instrumentos de evaluación de los cursos o de investigación a través de dispositivos.

8. Diseño y desarrollo de aulas virtuales para diferentes cursos, talleres.

9. Relación entre profesores de diversos centros (a través de redes y comunidades virtuales), compartir recursos $y$ experiencias, pasar informaciones, preguntas (Mata de López y Acevedo Blanco, 2010). 
Usar las TIC como ayuda al estudiante a buscar, analizar e interpretar críticamente información sobre el Ambiente y ampliar su potencial intelectual individual y colectivo. De esta manera, la sociedad cuenta con información renovable y necesaria para la toma de decisiones. En vista que las TIC forman parte del motor que impulsa a la sociedad, es indispensable conocer los distintos recursos que forman las TIC y sus aplicaciones, como son: estar al tanto de las posibilidades y limitaciones de las TIC, conocer cómo usarlas, y sobre todo apreciar el efecto de las TIC en el ámbito social.

\section{MÉTODO}

Esta es una revisión documental de los productos académicos de la UPEL-IPC declarados o no como desarrollos tecnológicos y cuyos temas se relacionan con las TIC. La metodología utilizada fue ejecutada en cinco fases: selección, revisión y registro de la información proporcionada en los I+D, aportes de experiencias de los autores y construcción de la teoría, de las cuales se presenta el resultado del análisis de los documentos.

La población estuvo conformada por todos los productos académicos relacionados con TIC (tesis, trabajos de grado, ponencias y artículos) y la muestra de los productos académicos relacionados con TIC (tesis, trabajos de grado y ponencias relacionados con investigación y desarrollo), Todo enmarcado en la observación directa participativa aunada a la experiencia de los autores en tutorías y asesorías de trabajos de grado y tesis doctorales, que hacen posible esta construcción. Esta investigación permite describir y hacer explícito el conocimiento almacenado relacionado con el área que nos ocupa.

\section{RESULTADOS Y DISCUSIÓN}

\section{Investigaciones $\quad y \quad$ Desarrollos relacionados con las TIC}

Hablar de los proyectos de desarrollo tecnológico registrados en la línea I+D o como parte de otras líneas que por desconocimiento $u$ otras causas es conversar acerca de proyectos cuyos áreas o campos temáticos son:

1. Innovación educativa y gestión de conocimiento.

2. Desarrollo de experiencias innovadoras y tecnología del aprendizaje y el conocimiento, tecnología empoderamiento y la participación.

3. Innovación, inteligencias múltiples, estilos de aprendizaje, gestión de conocimiento y pensamiento sistémico.

4. Innovación, evaluación y transformación educativa.

5. Desarrollo de experiencias innovadoras y de evaluación para la transformación del ámbito escolar.

6. Calidad de la educación: acciones para optimizar los procesos de enseñanza y aprendizaje.

7. Tecnología, productividad y servicio: niveles de eficiencia y productividad al servicio de la educación.

8. Desarrollo del recurso humano: formación inicial y permanente de estudiantes, docentes y otros profesionales.

9. Innovación educativa, aprendizaje invertido.

10. Autorregulación de Aprendizaje e Innovación educativa.

11. Producción de medios en diferentes formatos.

12. Aprendizaje computacional desconectado. 
13. Desarrollo y aprendizaje organizacional: proceso de planificación, organización y cambio en las organizaciones educativas y gestión de conocimiento.

Es de resaltar que Guzmán (2018) señaló que, en la UPEL, en Trabajos de Grado o Ascenso, ponencias, incluidos artículos en revistas arbitradas reconocidas nacional e internacionalmente, e incluso tesis doctorales y trabajo libres, se han realizado trabajos de desarrollo del estudiante, del Docente, desarrollo Organizacional y desarrollo Instruccional, en ellos se revisaron, investigaron y hecho propuestas para tratar de solucionar problemas relacionados con la organización sea escolar o no o cualesquiera de los elementos del sistema instruccional y las Tecnologías de Información y Comunicación, estos son:

1) TIC $y$ formación continua $y$ permanente del docente de diferentes niveles y especialidades $u$ otro profesional no docente. Expresan los problemas de formación y apropiación de las TIC en diferentes ámbitos.

2) TIC y estrategias en cualquiera de las teorías de enseñanza y aprendizaje. Incluyen problemas de formación $\mathrm{y}$ capacitación en estrategias basadas en el enfoque conductista proponiendo innovadoras enfocadas en las otras teorías. En algunos trabajos se sugieren estrategias tales como; enseñanza creativa, Aprendizaje en proyectos, Aprendizaje cruzado Aprendizaje Colaborativo, Gamificación, Storytelling, Aprendizaje Basado en retos, Aprendizaje invertido. Desarrollo de estrategias interactivas que promueven un aprendizaje autorregulado y significativo.

3) TIC y rol del docente. capacitación, actualización, profundización y formación en diferentes áreas del conocimiento con énfasis en uso y apropiación de las TIC tanto personal como profesional.

4) TIC y rol del estudiante formación en diferentes áreas del conocimiento con énfasis en uso y apropiación de las TIC tanto personal como profesional.

5) TIC y medios y recursos. Producción de medios digitales o no como parte de las soluciones factibles a problemas o como ayudas a los diseños de cursos, talleres diplomados. Unidades curriculares cuyo propósito son la alfabetización o formación en TIC para su uso como estudiante, ciudadano o futuro docente.

6) TIC y Educación en cualquier nivel o modalidad del. sistema educativo.

7) TIC, características y modalidades educativas (mixta, distancia o presencial y las plataformas para su uso)

8) TIC y sujetos digitales (generación $x, y, z$, nativos o inmigrantes, millenials) como parte del marco referencial pero no como objeto específico, desarrollan como se han comportado las TIC según las edades y características de estos sujetos.

9) Uso de TIC como medios y/o mediador del aprendizaje y de la investigación.

10) TIC y elementos directrices del sistema. Se basan en desarrollo de competencias, comunicativas, básicas, medias o avanzadas en TIC para docentes y otros profesionales.

11) Desarrollo y producción de medios (blogs, edublogs, ebook, podtcast, sitios web, audios, películas y videos entre otros).

12) Tecnologías de Información y Comunicación (TIC), Tecnologías para el Aprendizaje y el Conocimiento (TAC) y Tecnologías para el Empoderamiento y la Participación (TEP); conceptos, diferencias, semejanzas, ejemplos $\mathrm{y}$ sociedad. 
Si bien las TAC y TEP no han sido objeto de investigación en forma explícita se han obtenido evidencias de que se han desarrollado estas competencias tecnológicas para que los protagonistas del sistema interactúen, creen contenidos, medios, estrategias concibiendo una comunidad virtual e interactuando en una comunidad más participativa.

Por otra parte, se debe señalar que los actores del mundo de hoy, han visto cómo la tecnología se ha ido implantando en su rutina diaria, dando origen a un ciudadano que ya está inmerso en la tecnología quiéralo o no y requiere formación y entrenamiento para seguirle el ritmo a los avances tecnológicos. La UPEL no escapa a ello y valora que los Diseños Instruccionales se perfilen bajo una nueva visión de mundo y del conocimiento, tendente a construcciones socializadas con carácter interdisciplinario embebidas en las nuevas tendencias educativas y respondiendo a los cambios basados en las TIC. En consecuencia, es innegable que las instituciones están llamadas a conjugar esfuerzos para enfrentar tal desafío en la formación de futuras generaciones para la actual sociedad del conocimiento, y se aboquen a desarrollar esas competencias relacionadas con las TIC y el diseño instruccional (Suárez y De león, 2007 y 2008).

Po otra parte en relación con los elementos directrices (que puede aparecer bajo las formas: fines, propósitos, metas, contenidos (conceptuales, procedimentales o actitudinales), objetivos(generales, terminales, específicos, tareas y de contenidos y competencias) del sistema instruccional se puede referir que las TIC pueden verse como contenido, propósito, eje trasversal o curricular, un ejemplo de ello es en la UPEL que en el diseño 1996 se veía como parte de contenido de algunas asignaturas pedagógicas optativas y obligatorias y en el diseño 2015 se contemplan las TIC como eje curricular y deberían embeber a todas y cada una de las unidades curriculares de las diferentes especialidades de la carrera docente, de postgrado y de los diplomados ofrecidos por extensión.

Las TIC son requisito de ingreso a todos y cada uno de los postgrados que ofrece la Universidad Pedagógica Experimental Libertador y también están contempladas en las competencias específicas del área de Tecnociencia una de las tres áreas obligatorias en la extensión en el currículo que son requisitos de egreso en la formación de pregrado.

En relación con TIC y elementos directrices se han encontrado trabajos en cualesquiera niveles o modalidades del sistema educativo y en los cursos de adiestramiento de empresas, trabajos relacionados con Competencia Digital que se refiere al conjunto de capacidades, conocimientos, habilidades, destrezas $\mathrm{y}$ actitudes en relación al uso crítico, seguro y creativo de las tecnologías de la información y comunicación en la docencia,

Al revisar trabajos de grado tales como: Urbina, (2006), Rodríguez S. Z (2008), Rodríguez S. R (2008), Mendoza (2011), Rodríguez S. (2008, 2012), Guzmán y Rodríguez S. R. (2012), y Ramírez, (2018) y tesis doctorales tales como Rodríguez S. Z. (2016), Rodríguez S. R. (2016), López, (2016), De Marco, (2015) entre muchos otros consideran que el termino competencias son las capacidades con diferentes conocimientos, habilidades, pensamientos, carácter y valores de manera integral en las diferentes interacciones que tienen los seres humanos para la vida en el ámbito personal, social y laboral. Además, aplicar el enfoque de competencias es difícil porque tiene muchos significados, asimismo coinciden en señalar que hay competencias genéricas y específicas. Un ejemplo de eso son las competencias TIC que debe tener un docente de música, éste 
debe poseer competencias genéricas que debe poseer y aplicar además de eso, tener competencias propias de la especialidad en música (Solano, 2017).

Trabajos como el de Quintero J. Yoly (2017) referido al desarrollo de competencias en personal de una empresa de call center, el de Quintero J. Yuletzi, (2017) cuyo tema central es el desarrollo de competencias comunicativas; trabajos como el de Castillo (2019), Dilone (2019), Tovar (2019), Alcalá (2019), Hurtado (2019), Rodríguez S. R. y Guzmán, (2012), Rodríguez S. Z. y Guzmán, (2012), Rodríguez S. R. (2008), Rodríguez S. Z. (2008) cuyo objetivo el desarrollo de competencias en TIC en docentes y el de Delgado (2019) que desarrolla competencias en TIC en el personal de la biblioteca del estado Miranda demuestran que en los perfiles de las diferentes especialidades y en muchas otras profesiones se requiere la incursión y apropiación de las TIC.

Los hallazgos muestran que en estos trabajos se refieren los estándares TIC para docentes de la UNESCO $(2008,2016)$, además las qué presenta el proyecto Tunning y las de CINTERFOR que señalan que competencias de Secretary's Commission Achieving Necessary Skills (SCANS) (1991), y clasifica las competencias en Básicas y Transversales. Las competencias básicas se refieren a aquellas actitudes, destrezas y conocimientos más elementales que permiten ejercer al ser humano alguna actividad durante la cotidianidad o el día a día, mientras que las competencias transversales, son habilidades entendidas en todas sus dimensiones $\mathrm{y}$ pueden ser empleadas en cualquier ámbito laboral. Entre ellas la gestión de información: buscar y evaluar información, organizar, interpretar y comunicar y mantener sistemas de información, uso de computadores y el dominio tecnológico: seleccionar y aplicar tecnologías, dar mantenimiento y reparar equipos.
La información obtenida conduce a la relación entre la formación en TIC y su uso como estrategias para la praxis profesional, los hallazgos muestran que adecuada $y$ correcta formación, puede proporcionar múltiples ventajas a los docentes. Pues les permite acceder a recursos e información de manera inmediata; además, las actividades en el aula se podrían enriquecerse con actividades más motivadoras y lúdicas.

Entre las estrategias de enseñanza y aprendizaje con base en las TIC se encuentran: los foros, los grupos de discusión, los wikis, los chats, en los que los estudiantes realizan discusiones monitoreadas por el facilitador sobre los diferentes tópicos. Estas discusiones permiten a los participantes interactuar con los pares y los superiores, intercambiando conocimientos y años de experiencia que va a plasmar en sus comentarios, aportes, réplicas y conclusiones, lo cual implica, que todos aprenden de todo.

Los trabajos relacionados específicamente a las necesidades de los estudiantes que muchos "expertos" los asumen como milenials o nativos digitales porque han nacido en el auge de la tecnología digital, sin pensar en el contexto y la realidad que los rodea. Si ese estudiante no ha interactuado con las TIC así seas joven no eres ni nativo ni conoces de TIC, y ser de tercera edad tampoco te hace inmigrante, pero eso se tratara en otro momento. Los trabajos relacionados con los estudiantes tienen su origen en necesidades de formación o en formas de mediar mejor su aprendizaje, o de estrategias y modalidades de estudio en donde las TIC y el centro del proceso educativo, contenido como el aprendizaje invertido, autorregulación de aprendizaje (De Marco, (2015) y Poleo (2005) relacionada con características del educando ante el uso de las TIC y diferentes variables 
En una buena cantidad de trabajos publicados o no, generados, en diferentes asignaturas del postgrado y destinados a educación media o superior tienen como tema primordial la Producción de Medios. Digitales o no (ebook, sitios web, blog, contenido en formato CD ROM, podcast, videos, medios impresos, diseño de aulas virtuales, historietas entre otros. Ejemplos de estos productos son los trabajos de Cabezas y Guzmán (2012), De Marco y Guzmán (2005, 2008 y 2012), Vivas (2005). Ojeda (2003), Serrano (2003), Trejo y Marcano, (2013), Dilone y Delgado (2018), Guzmán (2013) que muestra medios digitales relacionados con educación ambiental, y reconoce que la búsqueda de información en los medios digitales es ponderada por encima de otras fuentes de información.

\section{CONCLUSIONES}

A manera de conclusión se puede señalar que las investigaciones y desarrollos tecnológicos educativos en la UPEL han sido exitosos y variados siempre con miras a solucionar problemas en las que proponen y se ejecutan competencias, estrategias, medios, cursos, unidades curriculares, diplomados, planes de formación, entre muchos otros. Este tipo de trabajo publicados o no le permiten a la educación solucionar muchos de los problemas en este caso relacionados con el uso y la apropiación de las TIC. Es importante desarrollar el pensamiento sistémico como forma de abordar la complejidad de la realidad imperante en estos días, para comprenderla, abordarla y transformarla.

\section{REFERENCIAS}

Alcalá A., (2019), Desarrollo de competencias para la enseñanza del turismo en los docentes de educación comercial de la Unidad Educativa Fe Y Alegría Virginia De Ruiz I - La Silsa. Trabajo de grado no publicada, UPEL-IPC
Álvarez, L, Arias A, Pérez U y Serrallé F. (2013). La historia de las ciencias en el desarrollo de competencias científicas. Enseñanza De Las Ciencias, Núm. 31.1 213-233

https://core.ac.uk/download/pdf/1332 7047.pdf [Consulta: 2018, septiembre $10]$.

Buch, T., (1996). La Tecnología, La Educación y Todo lo demás. Propuesta Educativa 7 6-15

Cabero, J. (1996 febrero) Nuevas Tecnologías, Comunicación Y Educación Universidad de Sevilla Edutec. núm. 1 [Revista Electrónica de Tecnología Educativa] disponible

en www.uib.es/depart/dcweb/revelec1.htl $\mathrm{m}$ [consultado enero 10, 2001].

Casado, D., Castro, S. y Guzmán, B. (2007). Las TIC En Los Procesos De Enseñanza Y Aprendizaje Revista Laurus [Revista en línea], Año 13, Número 23, Disponible: redalyc.uaemex.mx/pdf/761/76102311. pdf [Consulta: 2013, enero 15].

Castillo E. (2019). Competencias en el Uso Y Manejo De TIC De Los Docentes Del Departamento De Biología Y Química De La UPEL - IPC Trabajo de grado no publicado, UPEL-IPC.

Castro, S. y Guzmán, B. (2001). ¿Es la Tecnología útil en la Educación? Revista de Investigación, 49(1), 11-37.

Castro, S. y Guzmán, B. (2012). Materiales para el curso Tutoría I de la Maestría en educación. Mención Tecnología y Desarrollo de la Instrucción. Caracas. UPEL-IPC

Castro. S., (1993). Evaluación del curso de Nivelación en el área de Ciencias Naturales para estudiantes de nuevo ingreso del Instituto Pedagógico de Caracas. Trabajo de Ascenso no publicado. UPEL-IPC.

Castro. S. (2009). Políticas Educativas Basadas En La Gestión Del Conocimiento Para La Universidad Pedagógica Del Siglo XXI Tesis Doctoral no publicada UPEL-IPC. Caracas

Chadwick, C., (1975). ¿Qué es un tecnólogo educacional? Revista de Tecnología Educativa. 4 (1) p 7-9. 
Colciencias (1999 nov.). Guía de Proyectos de Innovación y Desarrollo Empresarial Disponible en http//.www.colciencias.gov.co/dic/guiai nnovacion.htm [consultado el 2019-0309].

De león, I, y Suárez, J. (2008). El Diseño Instruccional y Tecnologías de la Información y la Comunicación: Posibilidades y Limitaciones. Revista de Investigación, 32(65), 57-82. Disponible en

http://www.scielo.org.ve/scielo.php?scri pt=sci_arttext\&pid=S1010-

29142008000300005\&lng=es\&tlng=es [consultado el 2019-03-09].

De León, I. y Suárez, J. (2007). Diseño Instruccional y Tecnologías de la Información y la Comunicación. Algunas reflexiones. Revista de Investigación (61) pp. 13-33

De Marco, A. (2002). Las Nuevas Tecnologías Aplicadas En El Desarrollo Profesional Docente Del Departamento De Tecnología Educativa, Tesis de grado no publicada UPEL-IPC.

De Marco, A. y Guzmán, B. (2008). Diseño y Evaluación de un prototipo de CD rom para el curso Simulaciones y juegos instruccionales del Departamento de Tecnología Educativa en el Instituto Pedagógico de Caracas Revista de Investigación [Revista en línea] 65. Disponible:

http://www2.scielo.org.ve/pdf/ri/v32n 65/art08.pdf [Consulta: 2013, septiembre 10].

De Marco, A. y Guzmán, B. (2005). Evaluación Del Curso Simulaciones Y Juegos Instruccionales Del Departamento De Tecnología Educativa En El Instituto Pedagógico De Caracas. Trabajo de Ascenso no publicado. UPEL-IPC. Caracas.

De Marco, A., y Guzmán, B. (Enero - abril 2012). Un ambiente Web para la asignatura simulaciones y juegos instruccionales de la Universidad Pedagógica Experimental Libertador Revista de Investigación [Revista en línea] 75, (36). Disponible: http://www.scielo.org.ve/scielo.php?pid $=\mathrm{S} 1010$ -

29142012000100006\&script=sci_arttext [Consulta: 2019, septiembre 10].

Delgado N. (2019) competencias para el diseño de medios instruccionales apoyados en las TIC, dirigido a los funcionarios de la BPCE "CECILIO ACOSTA Trabajo de grado no publicado, UPEL-IPC

Dilone D. (2019). Integración De Las Tecnologías De Información Y Comunicación En La Educación Inicial Trabajo de grado no publicado, UPELIPC.

Dilone, D y Delgado, N. (2018). Producción de Material Instruccional en relación a los Peligros de Internet dirigido a los participantes del Proyecto de Servicio Comunitario Internet Seguro del Instituto Pedagógico de Caracas Trabajo de ascenso no publicado, UPEL-IPC

Escorsa, P y Valls J. (2001). Tecnología E Innovación en la Empresa. Dirección y Gestión. Alfaomega S: A Colombia

Göptepe, M. Özgüc, B. y Baray, M. (1989). Desing and implementation of a tool for teaching Programing. Computers Educ, 13 (2), 167-178.

Guzmán, B. (2005). Actitudes de los docentes ante las Tecnologías de Información y Comunicación Tesis Doctoral no publicada UPEL-IPC. Caracas

Guzmán, B. (2008). Los docentes de la Universidad Pedagógica Experimental Libertador - Instituto Pedagógico De Caracas ante las Tecnologías De Información $\quad \mathrm{Y}$ Comunicación Investigación y postgrado, ISSN 13160087, 1(23), págs. 37-72.

Guzmán, B. (2013). Evaluación del Curso Tecnología de Información y Comunicación y Educación Ambiental a Través de sus Productos. Trabajo de Ascenso para optar a la categoría de profesor Titular. UPEL-IPC

Guzmán, B. (septiembre 2018) TIC y Medios. 27 conferencia presentada en Encuentro Nacional de Profesores de Francés 
organizado y promovido por la Asociación Venezolana de Profesores de Francés. Pozo de Rosas, Miranda.

Hurtado Y. (2019). Desarrollo instruccional para la innovación educativa, basado en las TIC. Dirigido a los docentes de contabilidad del IPC proyecto de Trabajo de grado no publicado, UPEL-IPC.

Kaufman, R. (1983). Planificación de los sistemas educativos. 9na reimpresión. México. Editorial Trillas.

Kaufman, R. y Stone B. (1987). Guía Práctica para la Planificación en las Organizaciones. México. Editorial Trillas.

Mata de López A., Acevedo Blanco, A (2010) y La Actitud De Los Profesores Hacia El Uso De Las Tecnologías De La Información $\quad \mathrm{Y}$ Comunicación Investigación y Postgrado, Vol. 25 № 2 y 3, pp.143-180.

Mendoza, B. (2009). Competencias Tecnológicas para Docentes de la Unidad Educativa Centro Educacional de Invidentes "Francisco de Asís"-Fe y Alegría. Caso: Discapacitados Visuales Trabajo de grado no publicada, UPELIPC.

Moreno, F. (1978 octubre). Glosario Comentado sobre política Tecnológica Serie Material de apoyo para Programas de entrenamiento. CONICIT Caracas: Venezuela.

Müller, G. y Szczurek, M. (1989). Materiales para el curso Tutoría I de la Maestría en educación. Mención Tecnología y Desarrollo de la Instrucción. Caracas. UPEL-IPC.

Ojeda, N. (2003). Desarrollo de un software autoinstruccional en el área de informática para docentes de la III Etapa de Educación Básica, en la U.E. Instituto Filodidáctico Trabajo de grado no publicado, UPEL-IPC.

Oviedo, Y. (2010). Diseños Instruccionales Y Tecnologías De Información $Y$ Comunicación: Una Visión Del Docente De La UPEL-IPB, tesis doctoral no publicada Programa Interinstitucional De Doctorado En Educación (Pide) Barquisimeto Estado Lara.
Poleo, G. (1995). Desarrollo instruccional para propiciar la aplicación de Estrategia Cognitiva por docentes en alumnos de la Tercera etapa de E.B. Tesis de grado no publicada UPEL-IPC

Quintero J, Yoly. (2017). Competencias Laborales para Optimizar las Funciones de los Supervisores del Área del Call Center de la Empresa PRC333 C.A. Trabajo de grado no publicado, UPEL-IPC

Quintero J. Yuletzy, (2017). Desarrollo de la Competencia Comunicativa de los Estudiantes de la Asignatura Lengua Española en el Instituto Pedagógico de Caracas. Trabajo de grado no publicado, UPEL-IPC

Ramírez Y. (2013). Propuesta de un diseño instruccional en el uso de las TIC para docentes que laboran en el área de ciencias Trabajo de grado no publicado, UPEL-IPC

Rebolloso, R., (2000). La Globalización y las Nuevas tecnologías de Información Editorial Trillas México DF

Rodríguez S., R. (2008). Desarrollo de Competencias Asociadas a los Estudiantes que Ingresan a la Carrera de Profesor en Educación del Instituto Pedagógico de Miranda. Trabajo de Grado de Maestría no publicado, UPELIPC

Rodríguez S., R. (2016). Modelo Teórico para la Formación del Docente de Música Sustentado en el Desarrollo de Habilidades para el Arreglo Música. Tesis Doctoral no publicado, UPEL-IPC. Caracas.

Rodríguez S., Z. (2008). Desarrollo de Competencias Docentes a Través de la Incorporación del Uso de las Tecnologías de la Información y la Comunicación (TIC) en la Especialidad de Educación Musical. Trabajo de Ascenso UPEL-IPC.

Rodríguez S., Z. (2016). Corpus teórico para la formación del docente de música desde la perspectiva de los valores socioculturales. Caso UPEL. Tesis Doctoral no publicada. UPEL-IPC.

Rodríguez S., Z. y Guzmán B., (2012). Competencias en el uso de las TIC en profesores de la especialidad educación 
musical del Instituto Pedagógico José Manuel Siso Martínez. Revista de Investigación Año 2012, Vol. 36, Número 76

Serrano, R. (2003) Desarrollo autoinstruccional multimedia para el uso del programa micromundos, en el laboratorio de computación Trabajo de grado no publicado, UPEL-IPC

Seidner, C. (1976). enseñanza con simulaciones $y$ juegos tomado de Psychology of teaching methods. National Society for the study of ed. University de Chicago.

Solano I. (2017). Desarrollo de un Sistema Instruccional para el Estudio de la Guitarra Popular en la Escuela Superior de Música José Ángel Lamas. Trabajo de grado no publicada, UPEL-IPC

Stojanovic L. (septiembre- diciembre 2009). Tecnologías de comunicación e información en educación: Referentes para el análisis de entornos virtuales de enseñanza y aprendizaje Revista de Investigación № 68. Vol. 33 175https://www.researchgate.net/publicati on/44324498_Tecnologias_de_comunica cion_e_informacion_en_educacion_Refere ntes_para_el_analisis_de_entornos_virtua les_de_ensenanza_y_aprendizaje.

[Consulta: 2013, septiembre 10].

Szczurek, M. (1990, febrero). Tendencias actuales de la Tecnología educativa. Ponencia presentada en las Cuartas Jornadas de Tecnología educativa en Venezuela. Caracas. UCV

Tovar Z., (2019), Desarrollo Instruccional En Herramientas Tecnológicas Educativas Para Los Docentes De Educación Comercial De La Escuela Técnica Robinsoniana Y Zamorana Nacional Eduardo Meza Isturiz Trabajo de grado no publicado, UPEL-IPC

Trejo J, y Marcano N., (mayo- agosto 2013) Propuesta de innovación educativa mediante el uso de las TIC para la promoción de valores ambientales en la educación primaria venezolana Revista de Investigación № 79 Vol. 37

UNESCO. (2008). Estándares de Competencias en TIC para Docentes. [Documento en línea]. Disponible: http://www.eduteka.org/pdfdir/ UNESCOEstandaresDocentes.pdf [Consulta 2010, abril 2]

UNESCO. (2013). Enfoque Estratégico sobre TIC en Educación en América Latina y el Caribe. OREALC/UNESCO, Santiago

UNESCO. (2016). Competencias y Estándares TIC desde la dimensión pedagógica: Una perspectiva desde los niveles de apropiación de las TIC en la práctica educativa docente. Pontificia Universidad Javeriana. Cali, Colombia

UNESCO-IESALCCRES (2018). III Conferencia Regional de Educación Superior para América Latina y el Caribe CRES 20182028

UPEL (1996). Diseño curricular de la UPEL 1996. Documento base. Caracas

UPEL (2011). Diseño curricular de la UPEL 2011. Documento base. Caracas

Urbina, L. (2006). Las Competencias Básicas Asociadas Al Uso Instruccional De La Web Un Desarrollo Profesional Docente: Caso Unidad Educativa Fe Y Alegría "Virginia De Ruíz". Trabajo de grado no publicada UPEL-IPC

Vivas, M. (2005). Desarrollo Instruccional Orientado Al Uso De Las TIC En La Asignatura Estrategias $\mathrm{Y}$ Recursos Instruccionales Del Instituto Pedagógico De Caracas. Trabajo de grado no publicado. UPEL-IPC

Zabatta, V. (2008). Primeras Reflexiones sobre un Modelo General de Desarrollo Tecnológico. Investigación y Posgrado, 23(2), 433-446 